\title{
Identification of a novel gene fusion RNF213-SLC26A11 in chronic myeloid leukemia by RNA-Seq
}

\author{
JIAN-BO ZHOU, TING ZHANG, BEN-FANG WANG, HAI-ZHEN GAO and XIN XU \\ Department of Clinical Laboratories, Jiang Yin People's Hospital, Jiang Yin, Jiangsu 214400, P.R. China
}

Received June 29, 2012; Accepted October 25, 2012

DOI: $10.3892 / \mathrm{mmr} .2012 .1183$

\begin{abstract}
Chronic myeloid leukemia (CML) was the first hematological malignancy to be associated with a specific genetic lesion. The Philadelphia translocation, producing a BCR-ABL hybrid oncogene, is the most common mechanism of CML development. However, in the present study, b3a2, b2a2 and ela2 fusion junctions of the breakpoint cluster region (BCR)$\mathrm{V}$-abl Abelson murine leukemia viral oncogene homolog 1 (ABL) gene were not detected in patients diagnosed with CML three and four years previously. RNA-Seq technology, with an average coverage of $\sim 30$-fold, was used to detect gene fusion events in a patient with a 6-year history of CML, identified to be in the chronic phase of the disease. Using deFuse and TopHat-fusion programs with improved filtering methods, we identified two reliable gene fusions in a blood sample obtained from the CML patient, including extremely low expression levels of the classic BCR-ABL1 gene fusion. In addition, a novel gene fusion involving the ring finger protein 213 (RNF213)-solute carrier family 26, member 11 (SLC26A11) was identified and validated by reverse transcription polymerase chain reaction. Further bioinformatic analysis revealed that specific domains of SLC26A11 were damaged, which may affect the function of sulfate transportation of the normal gene. The present study demonstrated that, in specific cases, alternative gene fusions, besides BCR-ABL, may be associated with the development of CML.
\end{abstract}

\section{Introduction}

Chronic myeloid leukaemia (CML) is a clonal, myeloproliferative disease (1). The annual incidence of CML is $1-2$ cases per 100,000 individuals and the median age of presentation is 45-55 years, accounting for $15-20 \%$ of leukemia cases in adults (2). Based on clinical characteristics and laboratory

Correspondence to: Dr Jian-Bo Zhou, Department of Clinical Laboratories, Jiang Yin People's Hospital, Jiang Yin, Jiangsu 214400, P.R. China

E-mail: zhoujb9800@163.com

Key words: chronic myeloid leukemia, RNA sequencing, gene fusion, reverse transcription polymerase chain reaction, BCR-ABL findings, CML is often divided into three phases, chronic phase (CP), accelerated phase (AP) and blast phase (BP). Without intervention, CML typically begins in $\mathrm{CP}$ and over the course of several years progresses to AP, followed by BP. If administered early, drug treatment prevents this progression to a certain degree. A significant driver of progression from $\mathrm{CP}$ through AP and BP is the acquisition of additional chromosomal abnormalities to the Philadelphia ( $\mathrm{Ph}$ ) chromosome (2).

As the first consistent chromosomal abnormality associated with a specific type of leukemia, the identification of the Ph chromosome in 1960 by Nowell and Hungerford was a breakthrough in cancer biology (3). The $\mathrm{Ph}$ chromosome is the result of a $t(9 ; 22)$ reciprocal chromosomal translocation (4), involving the $\mathrm{V}$-abl Abelson murine leukemia viral oncogene homolog 1 (ABL) proto-oncogene normally located on chromosome 9 and the breakpoint cluster region (BCR) gene on chromosome 22 (5). The translocation results in deregulation of ABL tyrosine kinase activity and is defined as the pathogenetic principle (6). In addition to the classic BCR-ABL translocation in CML (7), a number of other gene fusions that contribute to oncogenesis have been reported in previous studies (8). Gene fusions are the result of aberrant chromosomal translocations that join together the exons of two unrelated genes, producing a chimeric mRNA transcript and protein (9). Previously, massively parallel RNA sequencing (RNA-Seq) was performed to identify gene fusions (10). RNA-Seq enables identification of gene fusions in individual cancer samples and facilitates comprehensive understanding of cellular transcriptomes $(11,12)$ through profiling the entire transcriptome at a level of detail unattainable by microarray (13).

In the present study, we performed RNA-Seq to detect gene fusions in a patient who did not present with common types of BCR-ABL gene fusions. Using an improved pipeline based on two tools for the detection of gene fusion, we identified several candidates of gene fusion in CP, including BCR-ABL1 and a novel gene fusion ring finger protein 213 (RNF213)-solute carrier family 26, member 11 (SLC26A11). Expression levels and functional domain change of the novel gene fusion were analyzed using a bioinformatic method and compared with genes involved in normal tissue funtion.

\section{Materials and methods}

Sample information. A single CML case from a patient of the Jiang Yin People's Hospital forms the basis of this report. The 
sample for gene fusion study was obtained in March 2011 and the patient was a Chinese male aged 78 years old. Clinical information was obtained from patient charts in the Jiang Yin People's Hospital. CML in CP was diagnosed in 2005 on the basis of a morphological study of bone marrow and peripheral blood specimens (total of $8.1 \%$ myeloblast and progranulocyte in blood smear with $\mathrm{WBC}=34.8 \times 10^{9} / 1, \mathrm{Hb}=62 \mathrm{~g} / \mathrm{l}$ and $\left.\mathrm{PLT}=529 \times 10^{9} / 1\right)$. In addition to increased myeloblast and progranulocyte levels, additional symptoms were identified, including blood dilution and splenomegaly demonstrated using ultrasonography. However, b3a 2 b2a 2 and ela 2 fusion junctions in the BCR-ABL gene were not detected in 2008. One year later, detection of the classic BCR-ABL breakpoint remained negative.

Written informed consent from the patient was obtained and the present study was reviewed and approved by the Clinical Laboratories Department of the People's Hospital in Jiang Yin City (Jiang Yin, China).

Library preparation. Total RNA was extracted from the peripheral blood using TRIzol according to the manufacturer's instructions (Invitrogen, Carlsbad, CA, USA). Following the TruSeq RNA SamplePrep Guide (Illumina, Inc., San Diego, CA, USA), the Illumina standard kit was used for mRNA-Seq sample preparation. Briefly, $10 \mu \mathrm{g}$ of total RNA extracted from the sample was used for polyA mRNA selection with polyT oligo-conjugated magnetic beads by two rounds of purification, followed by thermal mRNA fragmentation. Using reverse transcriptase (SuperScript II) and random primers, the fragmented mRNA was subjected to cDNA synthesis. Following this, cDNA was converted into double-stranded cDNA and after end repair [Klenow fragment, T4 polynucleotide kinase, T4 polymerase and 3-'A' add process (Klenow exo-fragment)] the product was ligated to Illumina Truseq adaptors. A 2\% agarose gel was used to perform size selection, which generated 380-bp cDNA libraries. Finally, the libraries were enriched through 15 cycles of PCR and purified with the QIAquick PCR purification kit (Qiagen, Hilden, Germany). Elution buffer was used to dilute the enriched libraries to a final concentration of $10 \mathrm{nM}$.

Primary analysis. Libraries from CML blood in CP were analyzed at a concentration of $11 \mathrm{pM}$ on a single Genome Analyzer IIx (GAIIx) lane using 115-bp sequencing. Raw RNA-Seq data were filtered using FASTx-tools (http:// hannonlab.cshl.edu/fastx_toolkit/) according to the following criteria: i) reads containing sequencing adaptors were removed; ii) nucleotides with a quality score $<20$ were trimmed from the end of the sequence; iii) reads $<50$ were discarded; and iv) artificial reads were removed. Following the filtering pipeline, all clean reads were trimmed to $90 \mathrm{bp}$ long due to 3' low quality base. A total of $3.2 \operatorname{Gbp}(3,193,208,820)$ of cleaned, paired-end reads was produced. Raw sequence data were submitted to the NCBI Short Read Archive (accession number, SRP011486).

Reads mapping and expression estimation. Clean and trimmed reads were aligned with the Ensembl $H$. sapiens reference genome (build GRCh37) using TopHat v1.3.3 (14). The program initially removes a portion of the reads based on quality information accompanying each read and then aligns the reads to the reference genome. TopHat allows multiple alignments per read (up to 20 by default) and a maximum of two mismatches when mapping the reads to the reference genome. The default parameters were used. Aligned read files were then processed by Cufflinks v1.2.1 (15), which measures the relative expression of the genes with the normalized RNA-Seq fragment counts. Fragments per kilobase of exon per million fragments mapped (FPKM) is used as the unit of measurement. A Bayesian inference method (16) was used to calculate confidence intervals for FPKM estimates. The reference GTF annotation file used in Cufflinks was downloaded from the Ensembl database (Homo_sapiens.GRCh37.63. gtf) (17). The gene expression data were submitted to the GEO database (accession ID, GSE36522).

Gene fusion prediction. Using TopHat, all filtered RNA-Seq reads were mapped to the reference transcriptome, which was downloaded from the Ensembl database (Homo_sapiens. GRCh37.63.cdna.all.fa). Read pairs mapping to the same transcripts were removed. Remaining reads were analyzed by two software packages, deFuse (deFuse-0.4.2) (18) and TopHat-Fusion (TopHat-Fusion-0.1.0) (19), to identify candidate gene fusions. The bowtie-index used in the TopHat-Fusion was downloaded from the TopHat homepage (H. sapiens UCSC hg19). The parameters of the TopHat-Fusion used were obtained from the 'Getting Started' (http://tophat-fusion. sourceforge.net/tutorial.html) tutorial. The deFuse parameters used were default.

Candidate gene fusion filtering. deFuse results were filtered according to McPherson et al (18) and Steidl et al (20). Two candidate gene fusions were obtained following the filtering pipeline. The TopHat-Fusion results were parsed by in-house Perl scripts and filtered according to the following pipeline: i) span reads were $>8$ reads; ii) ratio of against reads vs. span reads was $<0.5$; and iii) gene fusions involving ribosomal proteins or small nuclear ribosomal proteins were excluded. Following parsing, only one filtered candidate fusion remained. Filtered candidates detected simultaneously by deFuse and TopHat-Fusion were considered reliable candidate gene fusions. Following this filtering pipeline, a single reliable candidate, RNF213-SLC26A11, was obtained.

Improvement of filtering method. To reduce the false-positive rate and remove read-throughs, we improved the filtering standard by comparing the conditions of our filtering method with those of the final results. Two filtering standards of deFuse were modified: i) the number of splitr_count was revised according to the average coverage of the sequencing depth and ii) the events, which happened between 2 adjacent genes without any eversion or inversion, were considered to be produced by read-through. The detailed conditions of the 14 candidates are listed in Table I.

Gene fusion validation. To detect fusion transcripts, we designed a forward primer targeting the $5^{\prime}$ partner gene and reverse primer targeting the 3 ' partner. Primer pairs (Table II) for the coding exons of the fusion genes were generated using Primer 5 software (Premier Biosoft International, Palo Alto, 


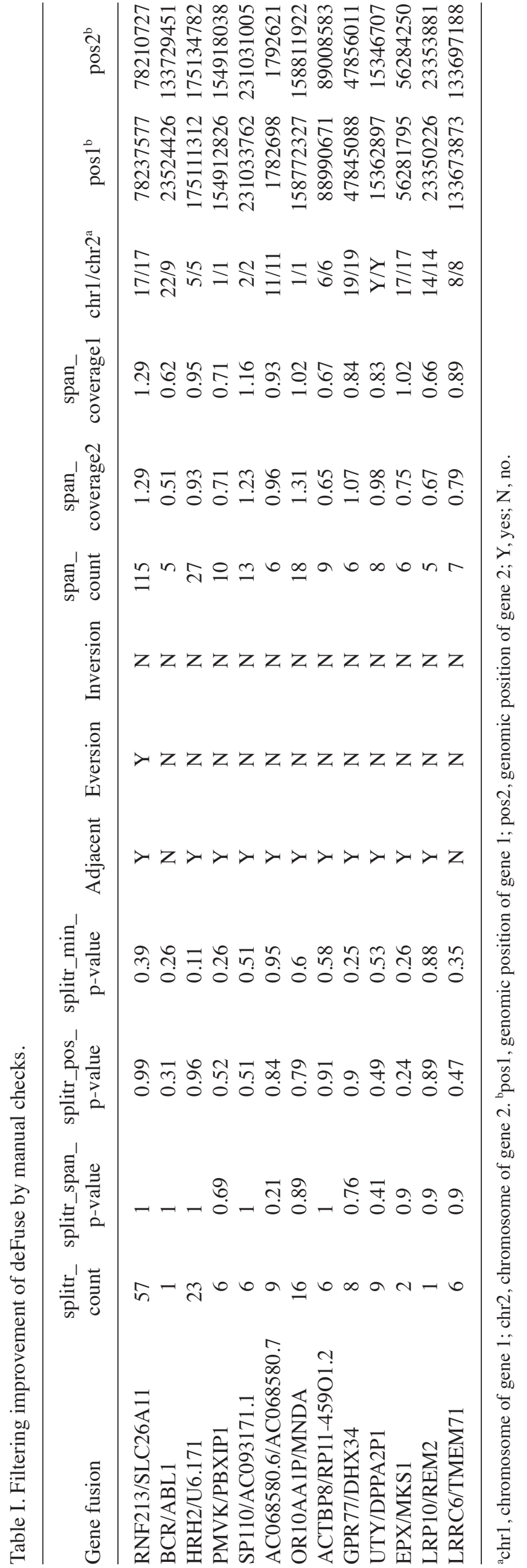

Table II. Primer pairs designed for detecting gene fusion.

\begin{tabular}{ll}
\hline Gene name & \multicolumn{1}{c}{ Primers (5'-3') } \\
\hline RNF213 & GACTCCTGCTCTTGCTTCTGG \\
SLC26A11 & ATCGTCCCGTTGGCTGTG
\end{tabular}

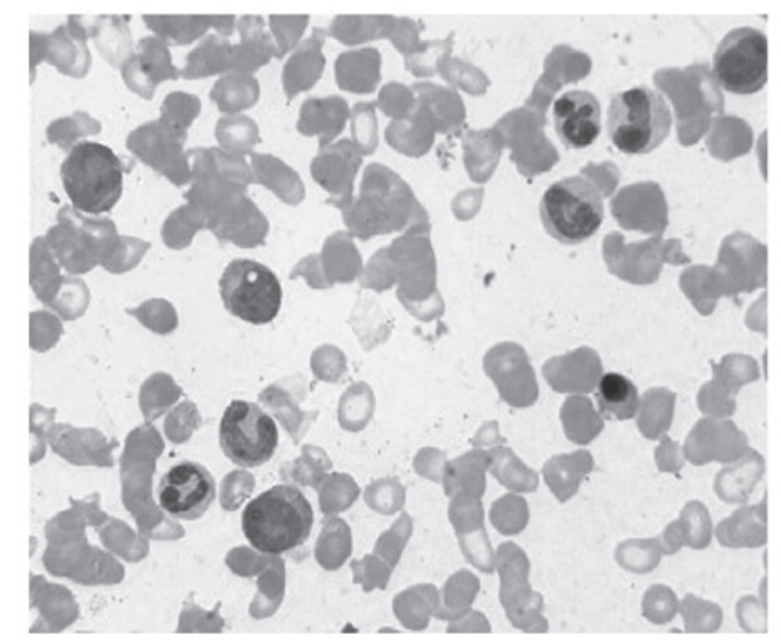

Figure 1. Peripheral blood smear obtained from the chronic myeloid leukemia patient $\left(\mathrm{WBC}=34.8 \times 10^{9} / 1\right.$; Wright-Giemsa stain; magnification, $\mathrm{x} 100$ ).

CA, USA) and the PCR volume comprised $20 \mu 1$ sample, $2 \mu 1$ 10X PCR buffer, $2 \mu \mathrm{l}$ cDNA template, $0.4 \mu \mathrm{l}$ dNTP, $0.4 \mu \mathrm{l} \mathrm{Taq}$ enzyme (Genscript, Piscataway, NJ, USA) and $0.2 \mathrm{pmol} / \mu 1$ each oligonucleotide. PCR was performed using the following procedure: $95^{\circ} \mathrm{C}$ for $2 \mathrm{~min}, 35$ cycles of $95^{\circ} \mathrm{C}$ for $15 \mathrm{sec}, 62^{\circ} \mathrm{C}$ for $20 \mathrm{sec}$ and $72^{\circ} \mathrm{C}$ for $20 \mathrm{sec}$, followed by $72^{\circ} \mathrm{C}$ for $2 \mathrm{~min}$. The presence of the fusion gene in the CML blood sample was confirmed. PCR products of the fusion gene were cloned in the pGEM $^{\circledR}$-T Easy Vector (Promega Corp., Madison, WI, USA) and then sequenced with the T7 primer using an Applied Biosystems 3730 DNA Analyzer (Life Technologies Corporation, USA).

Gene fusion visualization. Views of the candidate gene fusions were generated by running CIRCOS (available at http://circos. $\mathrm{ca} /$ ). Reads coverage of the candidate gene fusions in the sample were observed in the Integrative Genomics Viewer (IGV).

\section{Results}

Characterization of sequencing and mapping. CML blood in $\mathrm{CP}$ was obtained from a male patient of 78 years old. The peripheral blood smear is demonstrated in Fig. 1. Massively parallel paired end cDNA sequencing was performed using the Illumina Genome Analyzer IIx. In total, 43.6 million raw reads were produced. Following filtering and trimming, 35.5 million clean reads were obtained. All reads were aligned to the reference genome by TopHat. In summary, 15.3 million read pairs were uniquely aligned. Reads (74\%) were mapped to Ensembl reference genes. The average 
A
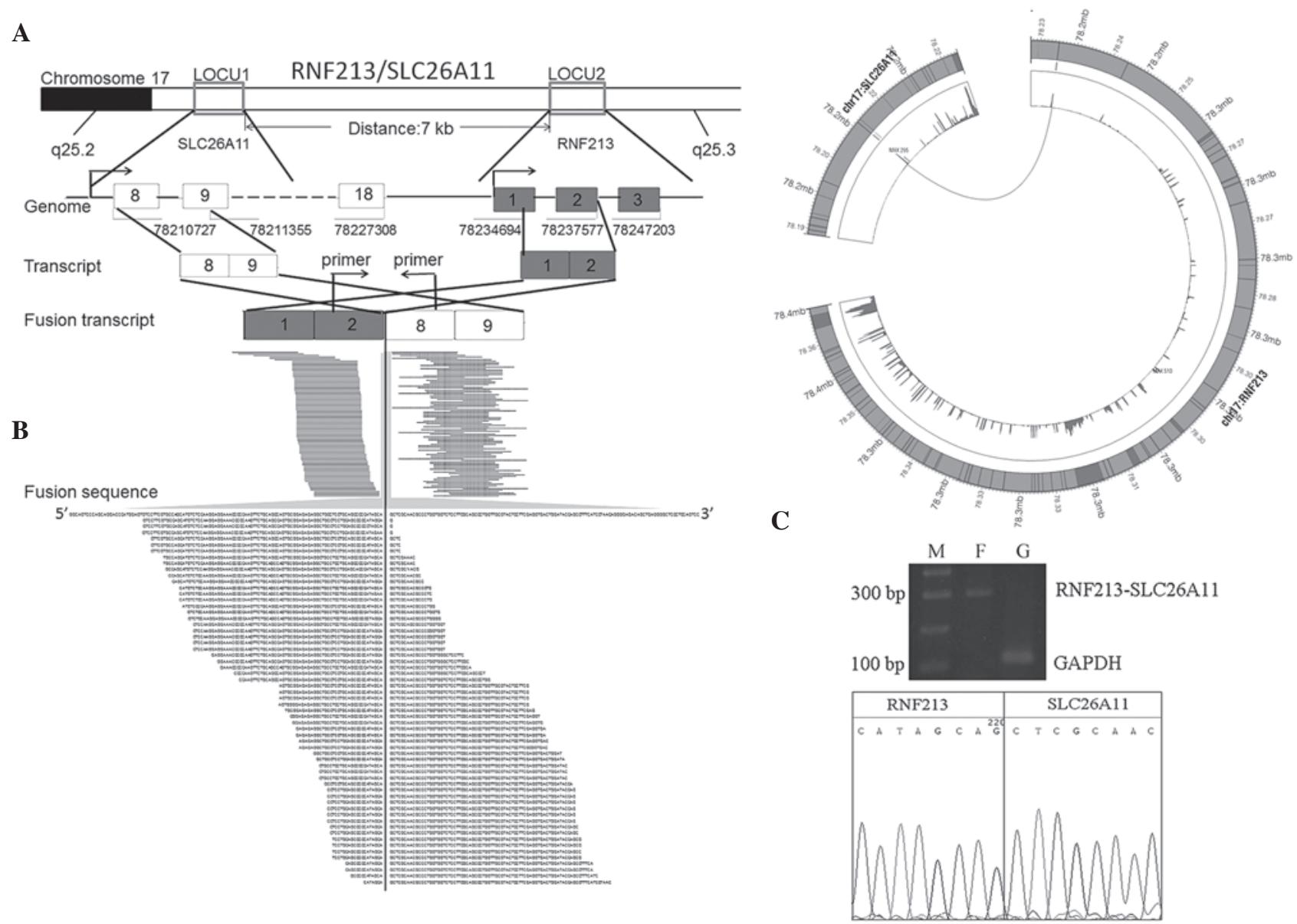

Figure 2. Molecular characterization of the gene fusion RNF213-SLC26A11 discovered in chronic myeloid leukemia. (A) Schematic of RNF213-SLC26A11 fusion on chromosome 17. The distance between these 2 loci is approximately $7 \mathrm{~kb}$. The second intron of RNF213 is fused with the eighth exon of SLC26A11. In the upper panel, 99 mate-pair sequences are shown aligning on either side of the breakpoint (pairing RNF213 and SLC26A11). The genomic coordinates of the exon boundaries are given. In the lower panel, 61 split-reads are depicted lying on the breakpoint (the first line, merged reference sequence of RNF213 and SLC26A11); (B) Genomic structure and reads coverage of RNF213 and SLC26A11. Selected chromosomes involved in the fusion events are drawn to scale around the rim of the circle and data are plotted on these coordinates in the outer ring. The inner ring displays reads coverage data inferred from whole transcriptome sequencing. The line links the 2 fusion points. MAX, maximum number of reads mapped to the region; (C) RT-PCR with the sequencing results of the fusion transcript in the sample..PCR primer is marked in (A). M, 100 bp ladder as marker; F, gene fusion RNF213-SLC26A11; G, GAPDH as control.

coverage of the sequencing depth was $>30$-fold of the human transcriptome. Only $4 \%$ reads were mapped to the $H$. sapiens mitochondrial genome. Raw 115-bp long reads from a single lane on the platform of Genome Analyzer IIx (GAIIx) was analysed. In addition, $20.89 \%(7,413,014)$ reads were splice junction reads. All observations were indicative of a correctly constructed library.

Analysis of gene expression. To measure gene expression in the sample, we utilized Cufflinks to estimate gene expression. Normalized expression levels of each gene were measured in FPKM. By selecting genes with an FPKM value $>1,22,607$ expressed genes were detected in the sample, including the majority of annotated reference genes in H. sapiens.

Prediction of gene fusion events. Two algorithms, including deFuse and TopHat-Fusion, were used to detect gene fusion events in the sample. Following removal of read pairs mapping to the same genes, 5.4 million $(\sim 30.9 \%, 5.4 / 17.7)$ reads pairs were obtained for gene fusion event prediction. Several gene fusion candidates were discovered, the majority of which were located within the chromosome. Following a manual check of the raw results produced by deFuse, unreliable results were removed. The filtered results of TopHat-Fusion and deFuse were combined to produce 14 gene fusion candidates (Table I). Among the candidates, one gene fusion event RNF213-SLC26A11 was detected by deFuse and TopHatFusion with a 3-bp breakpoint shift.

Improvement of filtering gene fusion. The majority of candidate gene fusions were identified within the same chromosome (Table I). Using manual checks and selection, we identified that the majority of fusion events occurred between adjacent genes on the same chromosome, which were usually regarded as read-through (21), while only two candidates, including RNF213-SLC26A11 and BCR-ABL1, were the most reliable gene fusion pairs. BCR-ABL is a well-documented gene fusion in CML and is used as a standard method for CML diagnosis (22). The RNF213-SLC26A11 fusion has not been previously identified in CML. In the present study, this novel fusion was further validated by reverse transcription polymerase chain reaction (RT-PCR) and the function of 
A

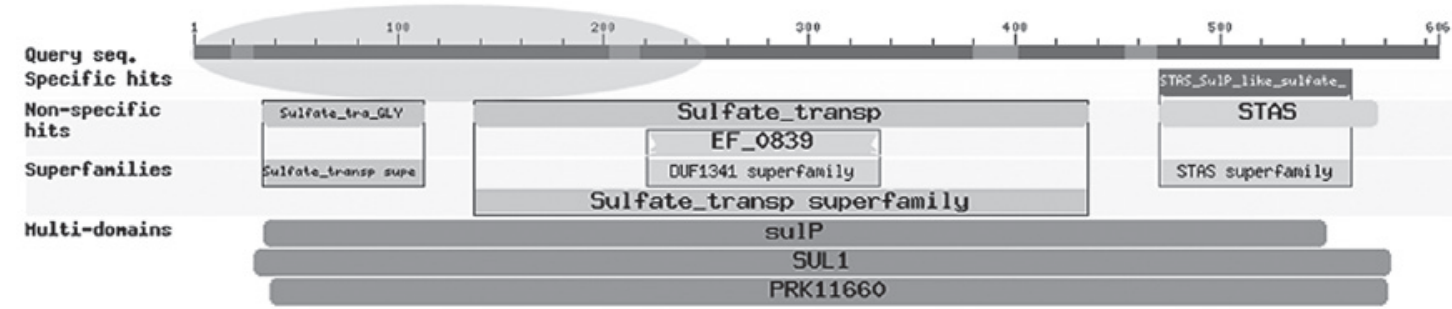

B

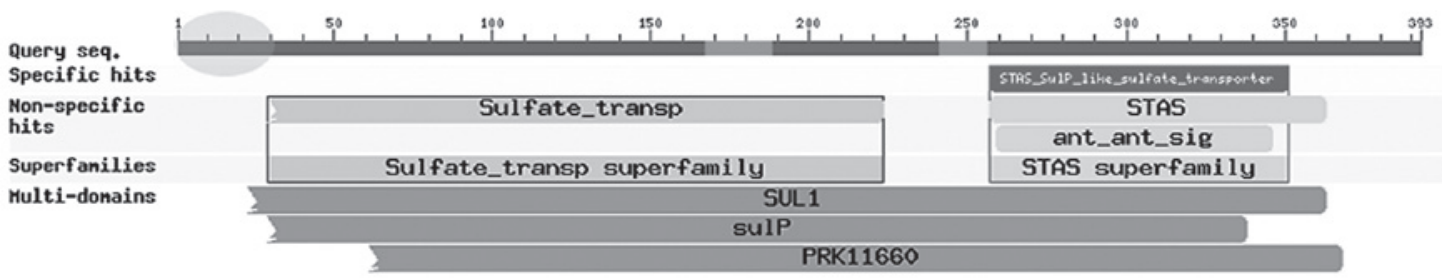

Figure 3. Prediction of protein domain. (A) Predicted protein domains of SLC26A11 under normal conditions. Oval-shaped region on the ruler represents the previous 7 exons of SLC26A11; (B) Predicted protein domains of RNF213-SLC26A11 in the CML sample, the oval-shaped region on the ruler represents the previous 2 exons of RNF213.

RNF213-SLC26A11 was predicted using bioinformatic methods.

$R T-P C R$ validation of RNF213-SLC26A11. To experimentally confirm the novel gene fusion identified by RNA-Seq, the expression level of RNF213-SLC26A11 in the sample was validated by RT-PCR. A primer pair, located at the second intron of RNF213 (5'-GACTCCTGCTCTTGCTTC TGG-3') and the 8th exon region of SLC26A11 (5'-ATCGTC CCGTTGGCTGTG-3') was designed. The results confirmed the existence of the fusion event in the sample (Fig. 2C), consistent with conclusions based on RNA-Seq analysis.

Identification of novel gene fusion RNF213-SLC26A11. As demonstrated in Fig. 2A, RNF213 and SLC26A11 are adjacent gene pairs located in chromosome 17 separated by $7 \mathrm{kbp}$. The genes are transcribed in the same direction in wild-type individuals. In the present sample, we observed a fusion between the second intron of RNF213 and the eighth exon of SLC26A11, generating a chimeric RNF213-SLC26A11 transcript, which has not previously been identified.

Analysis of reads coverage for RNF213 and SLC26A11. Analysis of reads coverage of RNF213 and SLC26A11 demonstrated that each exon of RNF213 was expressed at normal levels and the first 7 exons of SLC26A11 were expressed at extremely low levels (Fig. 2B), indicating that i) the RNF213-SLC26A11 fusion may occur in the majority of cells or ii) the fusion event altered expression of SLC26A11 using the promoter of RNF213, largely caused by the duplication of the first 2 exons of RNF213. RNF213 was normally expressed. In addition, compared with domains of the whole SLC26A11 gene under normal conditions (Fig. 3A), specific domains of the gene SLC26A11 in the chimeric transcript, including Sulfate_tra_GLY (pfam13792), EF_0839 (TIGR03581), sulfate_transp (pfam00916), sulP (TIGR00815), SUL1 (COG0659) and PRK11660 (PRK11660), were partially damaged (Fig. 3B). These observations indicate that the normal

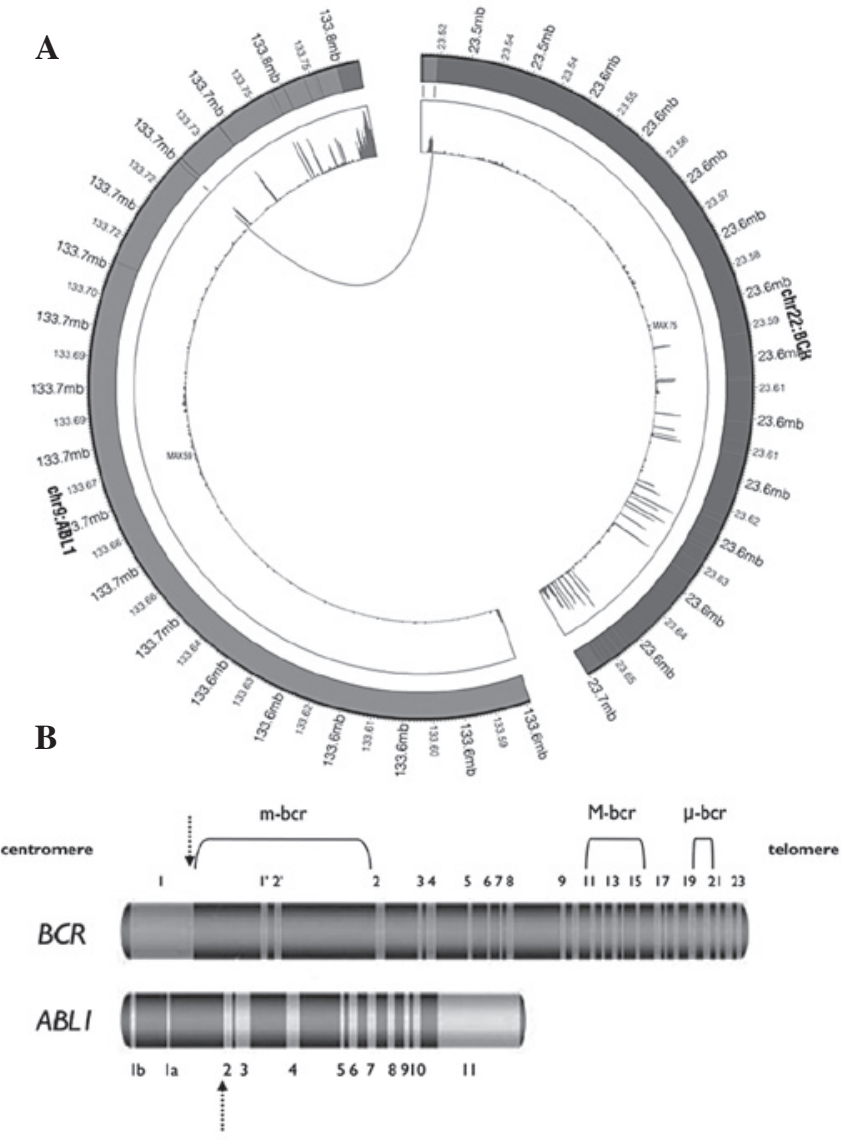

Figure 4. Molecular characterization of the recurrent gene fusion BCR-ABL1 discovered in chronic myeloid leukemia. (A) Genomic structure and reads coverage of BCR and ABL1; (B) schematic of the breakpoints of normal $\mathrm{BCR}$ and ABL1 genes.

function of RNF213 did not appear to be affected, while loss of function in SLC26A11 was identified. However, further investigation is required to understand the specific mechanism and functional consequence of this fusion event. 
Identification of recurrent gene fusion. In addition to the novel gene fusion, we identified another gene fusion candidate, which had been previously identified in CML (23). As demonstrated in Fig. 4B, BCR is located in chromosome 22 while ABL1 is on chromosome 9. In the present sample, a fusion was identified between the first intron of $\mathrm{BCR}$ and the second exon of ABL1, generating a chimeric transcript BCR-ABL1 (Fig. 4). Deininger et al (24) previously reported that this fusion is a ela2 fusion junction and is extremely rare (25).

\section{Discussion}

A number of methods are currently available for the detection of gene fusions, including chromosome banding analysis (karyotyping) followed by fluorescence in situ hybridization studies and molecular analysis based on RT-PCR (26). The RNA-Seq method currently enables genome-wide identification of novel fusion transcripts at the highest resolution level $(8,27,28)$. The BCR-ABL fusion transcript is considered to be a major consequence of the $\mathrm{Ph}$ translocation in CML (29). In the present study, whole transcriptome high-throughput sequencing was performed to detect additional fusion events to BCR-ABL, consistent with clinical diagnosis of CML. Using an improved bioinformatics method and RT-PCR, we identified extremely low levels of BCR-ABL1 in an ela2-type sample (FPKM: BCR=20.28, ABL=9.80). An additional novel gene fusion was also discovered.

To obtain the most reliable gene fusion candidates in the blood sample obtained from a CML patient in CP, the filtering method of deFuse was improved. Sample analysis using deFuse and TopHat-Fusion methods identified several gene fusion candidates. TopHat-Fusion results were filtered, mapping reads were checked manually and ribosomes were removed from the raw results produced by deFuse to obtain 14 gene fusion candidates. However, the majority of candidates were identified within the chromosome. Following detailed observation in IGV and improvement of the filtering method of deFuse, two reliable gene fusions were identified, including the novel gene fusion RNF213-SLC26A11 and the recurrent gene fusion BCR-ABL1.

From analysis of the structure of the novel chimeric transcript RNF213-SLC26A11, we identified that SLC26A11 expression is largely mediated by promoter displacement of RNF213. SLC26A11 was initially characterized as a putative sulfate transporter predominately expressed in high endothelial venules. SLC26A11 is widely expressed and mRNA expression has been identified in the kidney, placenta and brain (30). The blood sample analyzed in the present study was obtained from a CML patient in CP. Expression of the first 7 exons of SLC26A11 in this sample was observed at extremely low levels (Fig. 2B). In addition, SLC26A11 has been demonstrated to be expressed in the normal blood tissue in acute myoleid leukemia (http://www.genecards.org/ cgi-bin/carddisp.pl?gene=SLC26A11; http://www.genecards . org/info.shtml\#expression_images). Furthermore, a number of domains identified in normal human samples of SLC26A11 were also identified to be damaged by the formation of the novel chimeric transcript RNF213-SLC26A11 (Fig. 3B), including Sulfate_tra_GLY, EF_0839, sulfate_transp, sulP, SUL1 and PRK11660. STAS_SulP_like_sulfate_transporter (cd07042) and STAS (pfam01740) were observed to be unaffected. The present results indicate that the sulfate transport function of SLC26A11 was weakened, while its function in transport activity regulation and general NTP binding were maintained following fusion.

To explore the correlation between the RNF213-SLC26A11 gene fusion and CML, the invidual functions of RNF213 and SLC26A11 under normal conditions were identified. RNF213 encodes a protein containing a C3HC4-type RING finger domain, a specialized type of Zn-finger that binds two atoms of zinc and is thought to be involved in mediating proteinprotein interactions. The protein also contains an AAA domain, which is associated with ATPase activity. RNF213 is a susceptibility gene for Moyamoya disease, a vascular disorder of intracranial arteries (31). In addition, the protein functions as a translocation partner in anaplastic large cell lymphoma and inflammatory myofibroblastic tumor cases. A $t(2 ; 17)$ (p23;q25) translocation has been identified with the anaplastic lymphoma kinase gene located on chromosome 2 and a $t(8 ; 17)$ (q24;q25) translocation has been identified with the MYC gene on chromosome 8 (31). SLC26A11 encodes a member of the solute linked carrier 26 family of anion exchangers. Members of this family of proteins are essential for a number of cellular functions, including homeostasis and intracellular electrolyte balance. The encoded protein is a sodium-independent sulfate transporter that is sensitive to the anion exchanger inhibitor 4,4'-diisothiocyanostilbene-2,2'-disulfonic acid (RefSeq, Oct 2009). These functions indicate that the novel gene fusion candidate RNF213-SLC26A11 commonly occurs and affects homeostasis to a limited degree only. At present, the impact of this fusion on CML remains unclear. The mechanism of this functional change and its affect on the blood during CML require further investigations.

In addition to identification of the novel gene fusion RNF213-SLC26A11, we identified the recurrent gene fusion BCR-ABL1 at extremely low levels of expression. Analysis of the structure of this gene fusion demonstrated that the transcript discovered in our sample exhibits a e1a2 fusion junction, producing a 190-kDa protein (P190BCR-ABL). P190BCR-ABL CML has been previously identified in only $1 \%$ of CML patients and this junction is correlated with poor response to therapy with tyrosine kinase inhibitors (TKIs), with few, usually short-lived responses. Individuals with this gene fusion must be identified as high-risk patients, monitored closely for efficacy during therapy with TKI and offered stem cell transplantation early if eligible for this procedure (25).

Following improvement of the filtering method, we analyzed RNA-Seq data and discovered two gene fusions in a blood sample obtained from a CML patient in $\mathrm{CP}$, including a novel gene fusion RNF213-SLC26A11 and the recurrent BCR-ABL1 fusion. In addition, the present study has demonstrated that the current filtering method of deFuse must be revised according to the average coverage of the sequencing depth. This is likely to enable the improved filtering method to be applied to gene fusion detection in other tumor tissues.

\section{References}

1. Frazer R, Irvine AE and McMullin MF: Chronic myeloid leukaemia in the 21st century. Ulster Med J 76: 8-17, 2007. 
2. Faderl S, Talpaz M, Estrov Z and Kantarjian HM: Chronic myelogenous leukemia: biology and therapy. Ann Intern Med 131: 207-219, 1999.

3. Nowefl PC and Hungerford DA: A minute chromosome in human chronic granulocytic leukemia. Science 142: 1497, 1960.

4. Rowley JD: Letter: A new consistent chromosomal abnormality in chronic myelogenous leukaemia identified by quinacrine fluorescence and Giemsa staining. Nature 243: 290-293, 1973.

5. Tefferi A, Bren GD, Wagner KV, Schaid DJ, Ash RC and Thibodeau SN: The location of the Philadelphia chromosomal breakpoint site and prognosis in chronic granulocytic leukemia. Leukemia 4: 839-842, 1990.

6. Lugo TG, Pendergast AM, Muller AJ and Witte ON: Tyrosine kinase activity and transformation potency of BCR-ABL oncogene products. Science 247: 1079-1082, 1990.

7. Shtivelman E, Lifshitz B, Gale RP and Canaani E: Fused transcript of abl and bcr genes in chronic myelogenous leukaemia. Nature 315: 550-554, 1985.

8. Mitelman F, Johansson B and Mertens F: The impact of translocations and gene fusions on cancer causation. Nat Rev Cancer 7: 233-245, 2007.

9. Ha KC, Lalonde E, Li L, et al: Identification of gene fusion transcripts by transcriptome sequencing in BRCA1-mutated breast cancers and cell lines. BMC Med Genomics 4: 75, 2011.

10. Maher CA, Kumar-Sinha C, Cao X, et al: Transcriptome sequencing to detect gene fusions in cancer. Nature 458: 97-101, 2009.

11. Tang F, Barbacioru C, Wang Y, et al: mRNA-Seq whole-transcriptome analysis of a single cell. Nat Methods 6: 377-382, 2009.

12. Mortazavi A, Williams BA, McCue K, Schaeffer L and Wold B: Mapping and quantifying mammalian transcriptomes by RNA-Seq. Nat Methods 5: 621-628, 2008.

13. Wang Z, Gerstein M and Snyder M: RNA-Seq: a revolutionary tool for transcriptomics. Nat Rev Genet 10: 57-63, 2009.

14. Trapnell C, Pachter L and Salzberg SL: TopHat: discovering splice junctions with RNA-Seq. Bioinformatics 25: 1105-1111, 2009.

15. Roberts A, Trapnell C, Donaghey J, Rinn JL and Pachter L: Improving RNA-Seq expression estimates by correcting for fragment bias. Genome Biol 12: R22, 2011.

16. Jiang $\mathrm{H}$ and Wong WH: Statistical inferences for isoform expression in RNA-Seq. Bioinformatics 25: 1026-1032, 2009.

17. Hubbard TJ, Aken BL, Ayling S, et al: Ensembl 2009. Nucleic Acids Res 37: D690-D697, 2009.
18. McPherson A, Hormozdiari F, Zayed A, et al: deFuse: an algorithm for gene fusion discovery in tumor RNA-Seq data. PLoS Comput Biol 7: e1001138, 2011.

19. Kim D and Salzberg SL: TopHat-Fusion: an algorithm for discovery of novel fusion transcripts. Genome Biol 12: R72, 2011.

20. Steidl C, Shah SP, Woolcock BW, et al: MHC class II transactivator CIITA is a recurrent gene fusion partner in lymphoid cancers. Nature 471: 377-381, 2011.

21. Nacu S, Yuan W, Kan Z, et al: Deep RNA sequencing analysis of readthrough gene fusions in human prostate adenocarcinoma and reference samples. BMC Med Genomics 4: 11, 2011.

22. Melo JV, Gordon DE, Cross NC and Goldman JM: The ABL-BCR fusion gene is expressed in chronic myeloid leukemia. Blood 81: 158-165, 1993.

23. Quintas-Cardama A and Cortes J: Molecular biology of BCR-ABL1-positive chronic myeloid leukemia. Blood 113: 1619-1630, 2009.

24. Deininger MW, Goldman JM and Melo JV: The molecular biology of chronic myeloid leukemia. Blood 96: 3343-3356, 2000.

25. Verma D, Kantarjian HM, Jones D, et al: Chronic myeloid leukemia (CML) with P190 BCR-ABL: analysis of characteristics, outcomes and prognostic significance. Blood 114: 2232-2235, 2009.

26. Skotheim RI, Thomassen GO, Eken M, et al: A universal assay for detection of oncogenic fusion transcripts by oligo microarray analysis. Mol Cancer 8: 5, 2009.

27. Chen W, Kalscheuer V, Tzschach A, et al: Mapping translocation breakpoints by next-generation sequencing. Genome Res 18: 1143-1149, 2008.

28. Campbell PJ, Stephens PJ, Pleasance ED, et al: Identification of somatically acquired rearrangements in cancer using genomewide massively parallel paired-end sequencing. Nat Genet 40: 722-729, 2008

29. Hermans A, Selleri L, Gow J and Grosveld GC: Absence of alternative splicing in BCR-ABL mRNA in chronic myeloid leukemia cell lines. Blood 72: 2066-2069, 1988.

30. Vincourt JB, Jullien D, Amalric F and Girard JP: Molecular and functional characterization of SLC26A11, a sodium-independent sulfate transporter from high endothelial venules. FASEB J 17: 890-892, 2003

31. Pruitt KD, Tatusova T and Maglott DR: NCBI Reference Sequence (RefSeq): a curated non-redundant sequence database of genomes, transcripts and proteins. Nucleic Acids Res 33: D501-504, 2005. 